\title{
Análise do perfil de interação medicamentosa de plantas medicinas com os medicamentos mais utilizado por idosos hipertensos
}

\author{
Analysis of the drug interaction profille of medicine plants with the drugs more used by \\ hypertensive elderly \\ Análisis del perfil de interacción de fármacos de las plantas de medicina con los fármacos más \\ utilizados por ancianos hipertensivos
}

Recebido: 21/03/2021 | Revisado: 28/03/2021 | Aceito: 09/04/2021 | Publicado: 22/04/2021

Hyan Ribeiro da Silva

ORCID: https://orcid.org/0000-0002-3812-846X Centro Universitário UniFacid, Brasil

E-mail: hyanribeiro16@outlook.com

Raimundo Flanklin de Oliveira Neto

ORCID: https://orcid.org/0000-0002-0039-7963

Centro Universitário UNINOVAFAPI, Brasil E-mail: flanklinnneto348@gmail.com

Samara Cristina Lima Sousa

ORCID: https://orcid.org/0000-0003-3224-6569

Universidade Estácio de Sá, Brasil

E-mail: samaracristina129@gmail.com

Nágila Iane Pacheco

ORCID: https://orcid.org/0000-0002-2836-1639 Centro Universitário UniFacid, Brasil E-mail: nagilaiane@hotmail.com

Maria Hillana Nunes

ORCID: https://orcid.org/0000-0001-8193-235X Centro Universitário UniFacid, Brasil

E-mail: mhnunes38@gmail.com

Nathalia da Silva Brito

ORCID: https://orcid.org/0000-0002-0424-6222

Centro Universitário UniFacid, Brasil

E-mail: nathalia_brito18@hotmail.com

Fernanda Cristina dos Santos Soares

ORCID: https://orcid.org/0000-0003-0360-9068

Instituto Brasil de Pós Graduação IBRAS, Brasil E-mail: fernanda-crys-@hotmail.com

José Chagas Pinheiro Neto

ORCID: https://orcid.org/0000-0002-6626-6122 Universidade Federal do Piauí, Brasil E-mail: jose.cpneto@hotmail.com

Rayssa Hellen Ferreira Costa

ORCID: https://orcid.org/0000-0002-0322-4064 Universidade de Brasília, Brasil E-mail: rayssahfe@gmail.com

Mateus Henrique de Almeida da Costa ORCID: https://orcid.org/0000-0002-9866-4547 Universidade Federal do Piauí, Brasil E-mail: mateushenrick69@gmail.com

Ivanira Vieira Loiola Coutinho

ORCID: https://orcid.org/0000-0003-1877-1885 Centro Universitário UniFacid, Brasil E-mail: ivanirafarma@gmail.com Matheus Sena Lira

ORCID: https://orcid.org/0000-0001-9000-9217

Centro Universitário Santo Agostinho, Brasil

E-mail: matheus.lira123@hotmail.com

Moema Silva Reis

ORCID: https://orcid.org/0000-0002-2432-6182 Universidade Estadual do Ceará, Brasil

E-mail:moema_@hotmail.com

Gerson Tavares Pessoa

ORCID: https://orcid.org/0000-0002-6285-404X Centro universitário Maurício de Nassau, Brasil E-mail: gersonpessoa@hotmail.com 


\begin{abstract}
Resumo
As plantas com propriedades terapêuticas são utilizadas pelo homem ao longo de toda história da humanidade no tratamento e cura de enfermidades. O presente estudo trata-se de uma revisão sistemática de meta-análise realizada por meio de busca nas bases de dados: Lilacs, Google Acadêmico e Pubmed. O presente trabalho tem como objetivo geral realizar uma revisão sistemática analisando o perfil de interação medicamentosa de plantas medicinas com os medicamentos mais utilizado por idosos com pressão arterial e como objetivos específicos: descrever o uso de plantas medicinais por idosos; verificando o uso de plantas medicinais na hipertensão arterial. Diante o estudo fica evidente que os idosos fazem uso de plantas medicinais, com a finalidade de prevenir ou tratar alguma doença. Mesmo possuindo o conhecimento empírico sobre o uso destas, consomem pela automedicação, acreditando no poder da prevenção, tratamento e cura de doenças.
\end{abstract}

Palavras-chave: Interação; Plantas medicinais; Idoso; Pressão arterial.

\begin{abstract}
Plants with therapeutic properties are used by man throughout the history of humanity in the treatment and cure of diseases. The present study is a systematic review of meta-analysis carried out by searching the databases: Lilacs, Google Scholar and Pubmed. The present work has as its general objective to carry out a systematic review analyzing the profile of medicinal plant medicine interaction with the drugs most used by elderly people with blood pressure and as specific objectives: describe the use of medicinal plants by the elderly; checking the use of medicinal plants in high blood pressure. In view of the study, it is evident that the elderly make use of medicinal plants in order to prevent or treat any disease. Even having empirical knowledge about their use, they consume through self-medication, believing in the power of prevention, treatment and cure of diseases.
\end{abstract}

Keywords: Interaction; Medicinal plants; Elderly; Blood pressure.

\title{
Resumen
}

Las plantas con propiedades terapéuticas son utilizadas por el hombre a lo largo de la historia de la humanidad en el tratamiento y cura de enfermedades. El presente estudio es una revisión sistemática del metanálisis realizado mediante la búsqueda en las bases de datos: Lilacs, Google Scholar y Pubmed. El presente trabajo tiene como objetivo general llevar a cabo una revisión sistemática que analice el perfil de interacción de la medicina de plantas medicinales con los medicamentos más utilizados por las personas mayores con presión arterial y como objetivos específicos: describir el uso de plantas medicinales por parte de las personas mayores; comprobar el uso de plantas medicinales en la hipertensión arterial. Dado el estudio, es evidente que los ancianos usan plantas medicinales para prevenir o tratar cualquier enfermedad. Incluso teniendo conocimiento empírico sobre su uso, consumen a través de la automedicación, creyendo en el poder de prevención, tratamiento y cura de enfermedades.

Palabra clave: Interacción; Plantas medicinales; Ancianos; Presión arterial.

\section{Introdução}

A hipertensão arterial sistêmica (HAS) é apontada como fator de risco para a deterioração das funções cognitivas e também para a deterioração da qualidade de vida dos idosos, um dos maiores problemas de saúde pública. Ela se manifesta de forma multifatorial e é caracterizada por um nível elevado e rotineiro de pressão arterial sistólica acima de $140 \mathrm{~mm} \mathrm{Hg}$ ou pressão arterial diastólica acima de $90 \mathrm{~mm} \mathrm{Hg} 4,5$. Vale ressaltar que também é um importante fator de risco para morbimortalidade cardiovascular e, no Brasil, atinge 25\% da população acima de 20 anos (Bezerra, et al. 2018).

Os principais fatores de risco identificados em estudos são a circunferência elevada da cintura, sobrepeso e consumo frequente de gordura saturada, além disso, os pacientes costumam ter maus hábitos, como fumar, beber álcool e não se exercitar regularmente (Focchesatto et al. 2015).

De acordo com Santos (2017), a hipertensão arterial é uma doença que atinge aproximadamente 30 milhões de brasileiros e cerca de 50\% destes não sabem que são hipertensos, por serem muitas vezes assintomáticos, sendo considerado importante fator de risco para as doenças cardiovasculares ateroscleróticas, incluindo acidente vascular encefálico (AVE), doenças coronarianas, insuficiência vascular periférica e cardíaca.

Nesse sentido tem se o envelhecimento como um processo natural, onde ocorrem transformações biológicas e psíquicas, com ampla repercussão na esfera das relações do indivíduo, até o momento irreversível e comportando-se diferentemente em cada ser humano. No entanto, uma parcela significativa da humanidade precisa ter uma consciência clara de que os seres humanos estão envelhecendo e isso não significa que com o acúmulo de anos eles são escravos e perdem todos os direitos, muito menos 
que sejam considerados cidadãos de segunda classe. Embora seja verdade que os idosos sempre têm uma solução para tudo, um conselho, não é menos verdade que eles precisam de compreensão, ajuda e cuidado daqueles ao seu redor (Lemos et al., 2016).

O processo de envelhecimento individual está associado a alterações na capacidade funcional que podem ser prejudicadas pela presença de doenças crônicas e podem ser debilitantes. Nesse sentido, os idosos constituem a faixa etária mais vulnerável (Buranello, et. al. 2015). O termo qualidade de vida se tornou alvo de muitos idosos e ganhou um sentido mais amplo, se baseando em três dogmas fundamentais: capacidade funcional, nível socioeconômico e satisfação. Ainda, podendo se relacionar a partir dos parâmetros: estado emocional, capacidade física, atividade intelectual, interação social e autoproteção de saúde (Santos et al, 2017).

Dentre os fatores de risco associados à fragilidade em idosos, destaca-se o uso de medicamentos. Cerca de 70\% a 90\% dos idosos usam pelo menos um medicamento por dia, com uma média de dois a cinco medicamentos prescritos por idosos (Bezerra, et al, 2016).

Ressalta-se que, nesse contexto, os medicamentos são atualmente uma importante estratégia terapêutica e são utilizados em larga escala. No entanto, existe um risco inerente à sua ação farmacológica ou ao processo de sua utilização que pode levar ao desenvolvimento de reações adversas a medicamentos (EAM). A interação medicamentosa pode ser um fator que contribui para o surgimento do IAM. A interação medicamentosa é definida como o fenômeno que ocorre quando os efeitos ou a farmacocinética de um medicamento são alterados após a administração anterior ou coadministração de um segundo medicamento, as combinações de medicamentos podem ser benéficas quando proporcionam um melhor efeito terapêutico ou reduzem a toxicidade, mas podem ser prejudiciais quando favorecem o aparecimento de efeitos colaterais ou reduzem o efeito de um ou ambos os medicamentos (Balen, et. al. 2017).

Apesar de todos os avanços da indústria farmacêutica, as plantas medicinais definidas como aquelas capazes de produzir princípios ativos que podem alterar o funcionamento de órgãos e sistemas; ao restaurar o equilíbrio orgânico ou a homeostase nas doenças, continuam a contribuir para o tratamento de várias doenças em diferentes partes do mundo (Silva, et. al. 2017).

Desde a antiguidade, o homem faz uso de plantas medicinais com a finalidade de prevenir e curar diversas doenças. Essa tradição iniciou-se na China há mais de 3000 anos a.C, prática essa que até hoje é utilizada pelo conhecimento herdado de seus antepassados. No Brasil, a utilização popular das plantas medicinais é originada dos povos indígenas, que as utilizavam tanto para fins terapêuticos, quanto para rituais religiosos. Os africanos juntamente com os índios e europeus, foram os responsáveis pela formação da base do conhecimento cultural e biológico acerca das plantas úteis no Brasil (Silva, 2015).

No entanto, algumas das substâncias ativas responsáveis pela atividade biológica das plantas e produtos derivados são desconhecidas e, devido à sua complexidade, aumenta a probabilidade de interações medicamentosas de natureza sintética com administração e utilização simultâneas, visto que existem vários estudos. que demonstram os efeitos tóxicos e possíveis interações de plantas medicinais e / ou fitoterápicos quando usados indiscriminadamente (Silva, et. al. 2018)

Como consequência, a maioria dos usuários de plantas medicinais não informa o médico sobre esse uso, aumentando o risco de interações e, consequentemente, um possível erro diagnóstico (Carneiro; Comarella, 2016).

O enfoque nos idosos portadores de hipertensão arterial justifica-se pelo fato de constituírem um grupo populacional que culturalmente recorrem ao uso de plantas medicinais, por acreditarem que não causam danos à saúde e a hipertensão tem destaque no idoso por ser uma das doenças mais frequentes. Porém, o consumo de determinada droga vegetal, pode ser um risco para o idoso devido às possíveis reações adversas provocadas. Assim, o uso de plantas medicinais como recurso 9 terapêutico necessita de cuidados especiais, como qualquer outro tipo de tratamento, necessitando de acompanhamento de um profissional da saúde (Badanai, 2015).

Considerando que os idosos são os principais usuários de plantas medicinais/fitoterápicos e que a maioria necessita de fazer uso diariamente de medicamentos convencionais, dessa forma o presente trabalho tem como objetivo geral realizar uma 
revisão sistemática analisando o perfil de interação medicamentosa de plantas medicinas com os medicamentos mais utilizado por idosos com pressão arterial e como objetivos específicos: descrever o uso de plantas medicinais por idosos; verificando o uso de plantas medicinais na hipertensão arterial.

\section{Metodologia}

O presente estudo trata-se de uma revisão sistemática de meta-análise realizada por meio de busca nas bases de dados: Lilacs, Google Acadêmico e Pubmed. A escolha dos artigos foi realizada após a leitura do título, resumo, e pôr fim a leitura na íntegra. A etapa da revisão bibliográfica deste estudo indica que o conceito chave que compõem o cerne do trabalho diz respeito à identificação do que seja, em termos sociológicos e legais.

As palavras chaves utilizadas para a busca nos bancos de dados seguiram a descrição dos termos DeCs (Descritores em Saúde) no idioma português de inglês respectivamente: Interação (Interaction), Plantas Medicinais (Medicinal Plants), Idoso (Elderly), Pressão Arterial (Blood Pressure).

Os critérios de exclusão foram monografias, livros, resumos em eventos, artigos que não atenderam a temática, revisões bibliográficas ou sistemáticas e artigos duplicados.

Adotou-se como critérios de inclusão artigos de ensaios clínicos, randomizados, e estudos de casos, artigos publicados nos últimos 5 anos (2015 a 2020), que tivessem os descritores pesquisados no título e/ou no resumo e artigos publicados na integra.

\subsection{Meta-Análise}

As análises estratégia foi realizada com auxílio do programa SPSS para Windows 10, para leitura e entendido para contemplar a temática do artigo, para o cálculo da probabilidade foi seguido o resultado obtido conforme fluxograma. 
Fluxograma 1. Análise de dados.

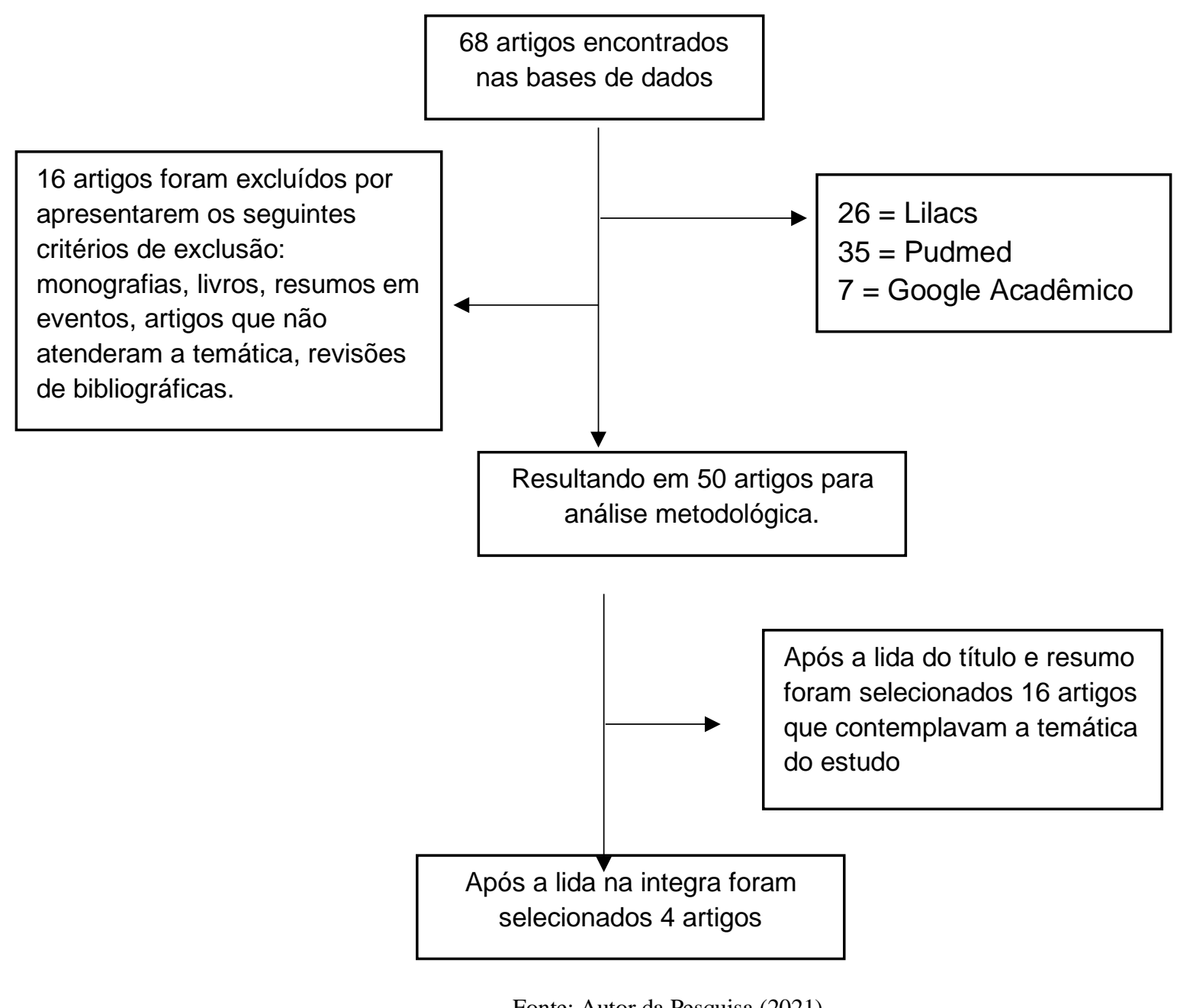

Fonte: Autor da Pesquisa (2021).

\section{Resultados e Discussão}

De acordo com as estratégias de busca foram encontradas um total de 68 artigos nas bases de dados selecionadas para a busca, sendo 35 artigos na base de dados Pubmed, 26 na base de dados Lilacs e 7 na base de dados Google Acadêmico, conforme Quadro 1. 
Quadro 1. Artigos Selecionados.

\begin{tabular}{|c|c|c|c|}
\hline Autor/Ano & Título & Amostra & Conclusão \\
\hline Bonil (2017) & $\begin{array}{l}\text { Plantas } \\
\text { Medicinais: } \\
\text { Benefícios } \\
\text { Malefícios }\end{array}$ & $\begin{array}{l}\text { Em amostras complexas como os } \\
\text { extratos vegetais, ressurgindo o } \\
\text { interesse por compostos de origem } \\
\text { vegetal que pudessem ser utilizados } \\
\text { como protótipos para o } \\
\text { desenvolvimento de novos fármacos. }\end{array}$ & $\begin{array}{l}\text { Pode-se concluir por meio deste trabalho } \\
\text { que, em princípio todas as plantas são } \\
\text { tóxicas, o que vai determinar se terão } \\
\text { efeito de remédio ou veneno é a dosagem. } \\
\text { O fato de a planta ser utilizada há muito } \\
\text { tempo popularmente não descarta a } \\
\text { possibilidade de ações tóxicas. }\end{array}$ \\
\hline Cascaes (2018) & $\begin{array}{l}\text { Perfil da } \\
\text { automedicação em } \\
\text { idosos } \\
\text { participantes de } \\
\text { grupos da terceira } \\
\text { idade }\end{array}$ & $\begin{array}{l}\text { Foi realizado um estudo transversal } \\
\text { baseado em entrevistas com idosos } \\
\text { participantes de grupos da terceira } \\
\text { idade, sendo solicitados além de dados } \\
\text { do perfil, informações sobre os } \\
\text { problemas de saúde, medicamentos } \\
\text { prescritos, prática da automedicação e } \\
\text { as alternativas utilizadas na mesma. }\end{array}$ & $\begin{array}{l}\text { Os idosos mesmo sendo uma população } \\
\text { polimedicada realizam a automedicação } \\
\text { sem a orientação de profissionais da saúde, } \\
\text { adotando principalmente plantas } \\
\text { medicinais e medicamentos de venda livre } \\
\text { por consideraram mais prático para o } \\
\text { manejo dos problemas de saúde que } \\
\text { identificam como simples. }\end{array}$ \\
\hline Feijo (2015) & \begin{tabular}{lr}
\multicolumn{2}{l}{ Plantas medicinais } \\
utilizadas & por \\
idosos & com \\
diagnóstico & com \\
Hipertensão & \\
Arterial & no \\
tratamento & dos \\
sintomas & da \\
doença. &
\end{tabular} & $\begin{array}{l}\text { O instrumento utilizado pela pesquisa } \\
\text { para a coleta dos dados foi um } \\
\text { questionário aplicado aos idosos que } \\
\text { haviam frequentado a UBS mais do } \\
\text { que duas vezes no último ano e } \\
\text { conseguiam comunicar-se } \\
\text { verbalmente. Este questionário } \\
\text { continha questões pré-codificadas, } \\
\text { sendo utilizadas para este trabalho as } \\
\text { questões que envolviam idosos } \\
\text { acometidos por DM, que referiram } \\
\text { utilizar plantas medicinais como } \\
\text { auxílio no tratamento dos sintomas da } \\
\text { doença. Na pesquisa foram abordados } \\
300 \text { idosos. Destes, } 92 \text { (30,7\%) } \\
\text { referiram diagnóstico de DM, sendo } \\
\text { que } 19 \text { citaram utilizar plantas } \\
\text { medicinais. }\end{array}$ & $\begin{array}{l}\text { Neste estudo identificou-se alguns } \\
\text { problemas em relação ao uso das plantas, } \\
\text { como a forma de preparo inadequada, a } \\
\text { procedência e o armazenamento } \\
\text { impróprio, podendo comprometer a } \\
\text { qualidade, propriedades funcionais e } \\
\text { benefícios à saúde. } \\
\text { É importante destacar que há necessidade } \\
\text { de cuidados ao utilizar uma planta } \\
\text { medicinal, pois nem todas têm o efeito } \\
\text { esperado por quem as utiliza. Isso pode } \\
\text { ocorrer devido à forma inadequada de } \\
\text { preparo ou ao fato de a planta não possuir } \\
\text { o princípio ativo para a ação desejada. }\end{array}$ \\
\hline Mauzalto (2018) & $\begin{array}{l}\text { O Controle da } \\
\text { Hipertensão } \\
\text { Arterial no Idoso. }\end{array}$ & $\begin{array}{l}\text { Trata-se de estudo transversal, de base } \\
\text { populacional, com amostra de } \\
\text { conglomerados, estratificada e em } \\
\text { múltiplos estágios. A análise dos } \\
\text { dados referentes aos } 426 \text { indivíduos } \\
\text { (sessenta anos e mais) levou em conta } \\
\text { o desenho amostral e o efeito do } \\
\text { delineamento. }\end{array}$ & $\begin{array}{l}\text { O presente artigo mostrou, em síntese, que } \\
\text { a hipertensão arterial é mais prevalente em } \\
\text { determinados subgrupos da população } \\
\text { como os idosos de menor escolaridade, } \\
\text { migrantes e com sobrepeso ou obesidade. } \\
\text { Ainda que as políticas públicas devam } \\
\text { contemplar a todos, atenção especial deve } \\
\text { ser voltada para os subgrupos mais } \\
\text { vulneráveis, tanto para as ações de } \\
\text { prevenção, de controle da hipertensão, } \\
\text { assim como para as de promoção à saúde. }\end{array}$ \\
\hline
\end{tabular}

Fonte: Autor da Pesquisa (2021).

Diante o estudo de Bonil (2017), as plantas com propriedades terapêuticas são utilizadas pelo homem ao longo de toda história da humanidade no tratamento e cura de enfermidades. É uma prática que nasceu provavelmente na pré-história, quando, a partir da observação do comportamento dos animais na cura de suas feridas e doenças, os homens descobriram as propriedades curativas das plantas e começaram a utilizá-las, levando ao acúmulo de conhecimentos empíricos que foram passados de geração para geração. 
Figura 1. Distribuição dos municípios que possuem ações/serviços com plantas medicinais e fitoterapia nas unidades federadas.

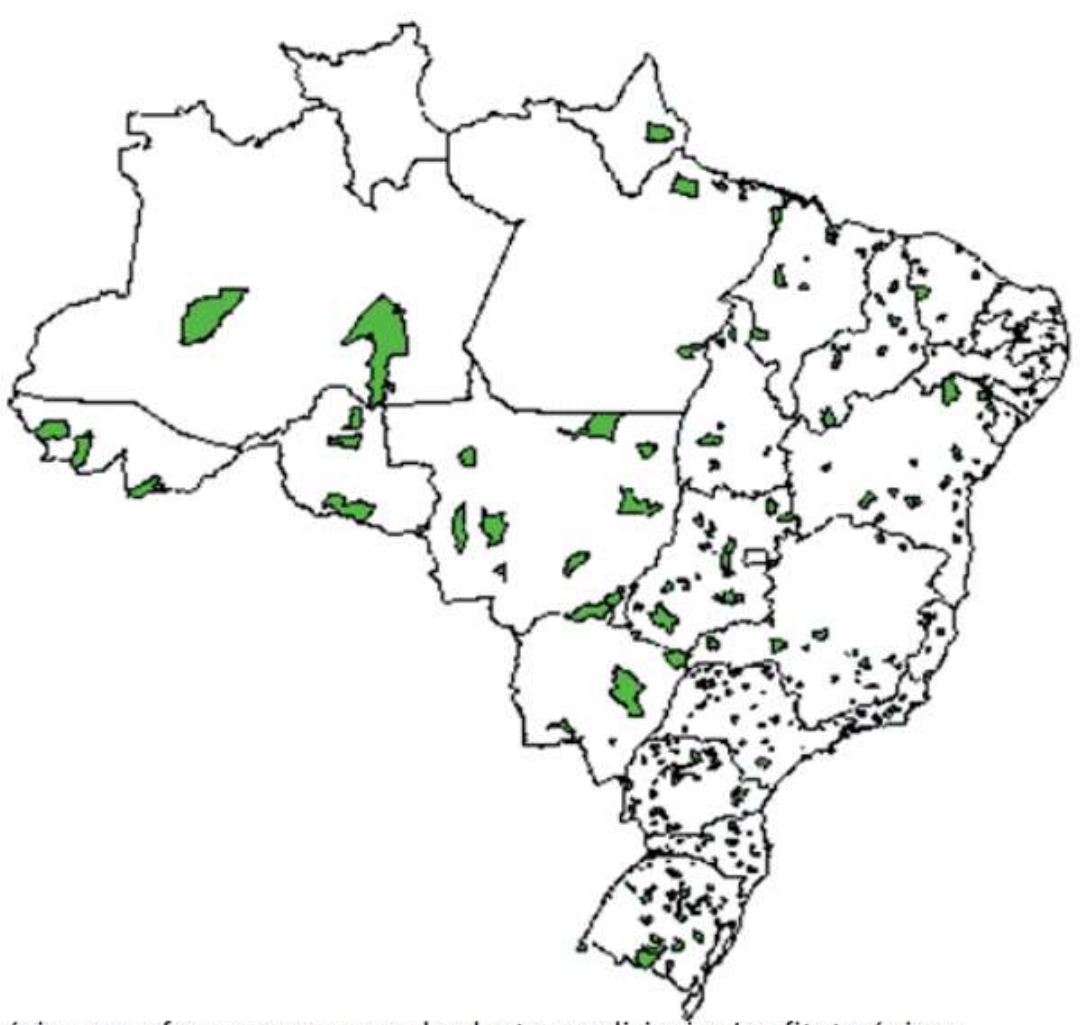

Municipios que oferecem o recurso de plantas medicinais e/ou fitoterápicos

Fonte: Bonil (2017).

Segundo Cascaes (2018), na Amazônia, as tradições populares de uso de plantas medicinais, representam um importante ponto de encontro entre permanências e rupturas culturais, estabelecidas desde os primeiros contatos intertribais e interétnicos e consolidadas no entrecruzamento das principais matrizes presentes no processo de formação do povo brasileiro (FIGURA 1).

Por isso, segundo Cascaes (2018), o uso de plantas medicinais e fitoterápicos no Brasil como medicamentos convencionais precisam apresentar critérios de qualidade, segurança e eficácia, através de levantamentos etno-farmacológicos, utilização, documentações tecno-científicas em estudos farmacológicos e toxicológicos pré-clínicos e clínicos.

De acordo com Feijo (2015), o autor acredita-se que esse cuidado realizado por meio de plantas medicinais seja favorável à saúde humana, desde que o usuário tenha conhecimento prévio de sua finalidade, riscos e benefícios. Além disso, o profissional que cuida do ser humano deve considerar tal prática de cuidado popular, viabilizando um cuidado singular, centrado em suas crenças, valores e estilo de vida conforme Quadro 2 abaixo.

Quadro 2. Descrição da relação entre planta medicinal e suas indicações para a pressão arterial.

\begin{tabular}{|c|c|c|c|}
\hline & Nome Popular/Científico & Indicaçao Popular & Indicação Científica \\
\hline 1 & Erva-cidreira (Lippia Alba) & Calmante & Calmante, espamolitica sauva \\
\hline 2 & Hortelã (Menta x villosa) & Calmante, digestão & Vermífuga. \\
\hline
\end{tabular}

Fonte: Adaptado de Feijo (2015).

Mauzalto (2018), afirma que a maioria dos idosos utilizam muitos medicamentos para tratar diversas doenças crônicas e mesmo com os avanços tecnológicos envolvendo a área da saúde, ainda fazem uso frequente de plantas medicinais, pois acreditam que por ser natural não possuem reações adversas, desconhecendo as possíveis interações que podem ocorrer. Ao longo do tempo têm sido registrados variados procedimentos clínicos tradicionais utilizando plantas medicinais. 
Diante do estudo de Cascaes (2018), um fato importante a ser destacado é que o uso de qualquer terapêutica em idosos requer maiores cuidados, pois os idosos estão em processo de degeneração orgânica, o que de certa forma dificulta o curso dos princípios ativos das ervas no organismo, além de muitos possuírem órgãos cujo funcionamento já não é o suficiente adequado, como é o caso do fígado e dos rins. Sendo assim, são necessários maiores cuidados.

Dessa forma Mauzalto (2018), afirma que as medidas não farmacológicas são experimentadas em primeiro lugar, especialmente nos casos brandos recém-detectados, caso as medidas forem ineficazes, o tratamento evolui de maneira progressiva para os agentes anti-hipertensivo. Algumas mudanças nos hábitos de vida como diminuir a quantidade de sal na preparação dos alimentos, evitar o saleiro à mesa, utilizar sal de ervas reduzir o consumo de alimentos industrializados, incluir frutas, verduras e cereais integrais na dieta diária; consumir alimentos com teor de gordura reduzido, uso de ervas medicinais, moderar o consumo de álcool.

Segundo Feijo (2015), as plantas produzem uma grande variedade de substâncias químicas que podem apresentar diversas atividades biológicas no organismo, podendo ser benéficas ou não. Vale ressaltar que a utilização de plantas na terapêutica e na alimentação deve ser restrita a plantas conhecidas e corretamente identificadas, pois podem ocorrer intoxicações com o uso de espécies vegetais (Figura 2).

Figura 2. Esquema para planta medicinal cutivada e disponibilizada na forma fresca (in natura).

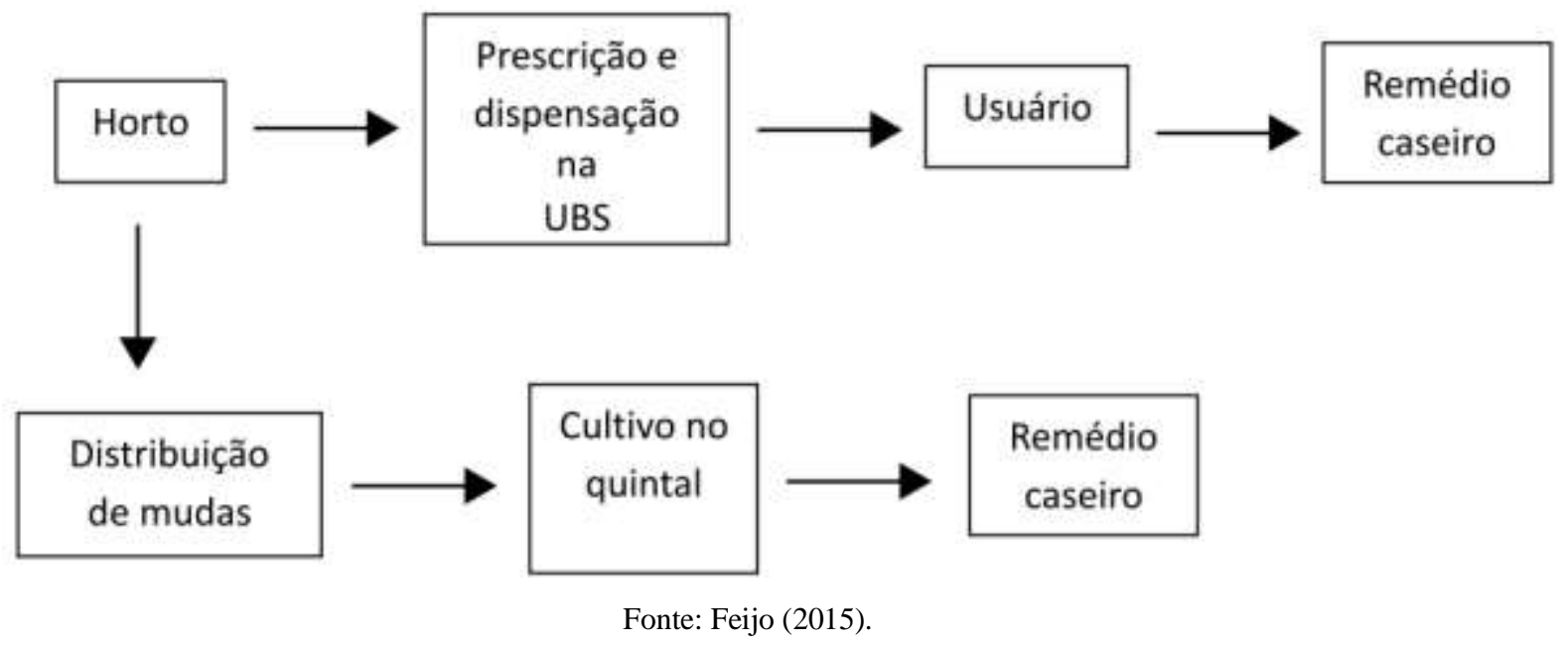

No estudo de Bonil (2017), foi verificado sobre o conceito de que "é natural e não faz mal", faz com que muitas pessoas façam uso baseando-se apenas em uma suposição de que não causariam dano, e assim não representariam perigo à saúde. Além dos problemas oriundos da interação medicamentosa, o mau uso de plantas medicinais pode ocasionar problemas à saúde que podem levar a internações hospitalares e até mesmo à morte, dependendo da forma de uso arterial.

Segundo Feijo (2015), ao avaliar as interações das plantas medicinais com medicamentos anti-hipertensivos as mais evidenciadas neste levantamento foram: Crataegus oxyacantha, Ginkgo biloba e Pausinystalia iohimbe. Porém outras plantas medicinais que tem seu efeito sobre a PA evidenciados através de estudos científicos também podem interagir com esta classe de medicamento, mas há necessidade de mais estudos relacionados a este assunto. O potencial de interação planta/medicamento vai depender, assim como o efeito da planta sobre a pressão arterial, da presença e mecanismo de ação de alguns metabólitos secundário.

Para Mauzalto (2018), a possível interação entre o Allium sativum e medicamento anti-hipertensivo, pode ser explicada pelo fato de que os compostos sulfurados do alho apresentam atividade in vitro vasodilatadora mediado pela liberação de óxido 
nítrico, potencializando o efeito hipotensivo do medicamento quando utilizado concomitantemente, pois com o aumento da vasodilatação o débito sanguíneo passa a ter uma melhor circulação vascular, reduzindo a pressão na parede dos vasos.

Ainda de acordo com o estudo de Mauzalto (2018), este mesmo mecanismo de interação pode ser atribuído a outras plantas medicinais com ação vasodilatadora. Já a planta Pausinystalia johimbe possui um alcalóide conhecido como ioimbina que tem propriedade antagonista e causa interação com anti-hipertensivos que agem neste mesmo local, antagonizando seus efeitos e aumentando a pressão arterial.

\section{Conclusão}

O uso de plantas medicinais é consequente de um conhecimento passado através de gerações no núcleo familiar, e nesse núcleo a figura da mulher tem um papel fundamental na manutenção e transmissão desse conhecimento. $\mathrm{O}$ fato dessas práticas complementares, já estarem inseridas no cotidiano, faz desses saberes algo importante para o cuidado de enfermagem, para que esse cuidado esteja adequado às características e necessidades da clientela e assim poder enlaçar o conhecimento popular ao científico.

Diante o estudo fica evidente que os idosos fazem uso de plantas medicinais, com a finalidade de prevenir ou tratar alguma doença. Mesmo possuindo o conhecimento empírico sobre o uso destas, consomem pela automedicação, acreditando no poder da prevenção, tratamento e cura de doenças. Além disso os autores afirmam os efeitos tóxicos de sua utilização inadequada, sendo necessário buscar cada vez mais estudos que verifique os riscos da utilização do uso inadequado.

\section{Referências}

Badanai, J. M. (2015). Utilização De Plantas Medicinais, Fitoterápicos e dos Potenciais Riscos de suas Interações com Medicamentos. Escola - São Caetano do Sul. 18 f. Monografia.

Balen, et. al. (2017). Interações medicamentosas potenciais entre medicamentos psicotrópicos dispensados. J Bras Psiquiatr. $2017 ; 66(3): 172-7$.

Bezerra, A.L.A. et al (2018). Perfil epidemiológico de idosos hipertensos no brasil: uma revisão integrativa. Rev Med (São Paulo). 2018 jan.-fev.;97(1):103-7.

Bezerra T.A et al (2016). Caracterização do uso de medicamentos entre idosos de uma unidade do Programa Saúde da Família. Cogitare Enferm. 2016;21(1):111. [ Links ]

Bonil, L. N., \& Bueno, S. M. (2017). Plantas Medicinais: Benefícios e Malefícios.

Buranello, et. al. (2015). Síndrome de fragilidade em idosos da comunidade: características socioeconômicas e de saúde: um estudo observacional. Medicina (Ribeirão Preto). 2015;48(5):431-9.

Carneiro; Comarella, (2016). Principais interações entre plantas medicinais e medicamentos. Revista Saúde e Desenvolvimento, 9(5). 4-19, 2016.

Cascaes, E. A. (2018). Perfil da automedicação em idosos participantes de grupos da terceira idade.

Feijo, A. M. et al. (2015). Plantas medicinais utilizadas por idosos com diagnóstico com Hipertensão Arterial no tratamento dos sintomas da doença. Rev. Bras. Plantas Med. 14(1), 50-56,

Focchesatto et al. (2015). Fatores de risco e proteção para o desenvolvimento de doenças crônicas em população idosa rural do Rio Grande do Sul. Rev Bras Geriatr Gerontol. 2015;18(4):779-95

Lemos, S. (2016). Envelhecimento ativo: uma política de saúde. Organização Pan-Americana da Saúde,

Mauzalto, A. C. M (2018). O Controle da Hipertensão Arterial no Idoso. 38f. Universidade Federal do Triângulo Mineiro.

Silva, B.B.M. et al (2018). Interação medicamentosa entre plantas medicinais/fitoterápicos e fármacos Congresso III CONBRACIS, 2018.

Silva, B. Q., \& Hahn, S. R (2015). Uso de plantas medicinais por indivíduos com hipertensão arterial sistêmica. R. Bras. Farm. Hosp. Serv. Saúde, 2(3), 36-40. Silva, et. al. (2017). A utilização de plantas medicinais e fitoterápicos em prol da saúde única cadernos acadêmicos 1(1).

Santos, S. R. et al (2017). Qualidade de vida do idoso na comunidade: aplicação da Escala de Flanargan. Revista Latino-americana de Enfermagem. 10(6), 757764. 